\title{
Le polymorphisme de coloration abdominale de Drosophila erecta est-il gouverné par un gène sélectivement neutre?
}

\author{
Véronique PAYANT \\ C.N.R.S., Laboratoire de Biologie et Génétique Evolutives \\ 91190 Gif-sur-Yvette, France
}

\begin{abstract}
Résumé
Les modalités du maintien du polymorphisme au locus de coloration abdominale chez Drosophila erecta ont été recherchées au laboratoire par l'analyse des composantes de la valeur sélective. Onze populations ont été suivies en cages pendant 30 générations à 4 températures. Quelle qu'ait été la fréquence allélique initiale, aucun équilibre de fréquence n'a été atteint. Les valeurs sélectives des différents génotypes aux stades larvaire et adulte ont été estimées, aucune différence nette n'a été mise en évidence. Le gène gouvernant la coloration abdominale dans cette espèce pourrait être neutre : cette éventualité est discutée.
\end{abstract}

Mots clés : polymorphisme, sélection, neutralité, D. erecta .

\section{Summary}

Is the polymorphism of abdomen pigmentation in Drosophila erecta under the influence of a neutral gene?

The components of fitness for the various genotypes at the locus controlling the abdomen pigmentation in $D$. erecta were investigated in order to explain the maintenance of polymorphism at this locus. Eleven experimental population cages, maintained at 4 different temperatures, were constructed starting with different initial allelic frequencies. Equilibrium was not reached after 30 generations. No difference appeared between genotypes concerning the larval or the adult fitnesses, which would possibly explain the maintenance of this polymorphism. The possibility of selective neutrality at this locus is discussed.

Key words : polymorphism, selection, neutrality, D. erecta.

\section{Introduction}

Peu d'exemples de polymorphisme morphologique naturel sont connus chez les drosophiles bien que de nombreux mutants aient été répertoriés dans ce groupe. L'une 
des premières espèces chez laquelle un polymorphisme fut décrit a été nommée, pour cette raison, Drosophila polymorpha (Dobzhansky \& Pavan, 1943). Ce polymorphisme concerne la coloration abdominale. Il a été décrit depuis chez diverses espèces de drosophiles, qui peuvent être groupées en deux catégories :

- celles qui présentent deux phénotypes distincts, sombre et clair : $D$. andalusiaca (BASDEN, 1957), D. rufa (Oshima, 1952), etc. Dans ce groupe le déterminisme génétique de ce caractère est en général monofactoriel et diallélique ;

- celles chez lesquelles il existe une variation continue entre deux phénotypes extrêmes : $D$. melanogaster, $D$. simulans, etc. Dans ce groupe l'expression du caractère varie en général avec la température d'élevage, les individus les plus sombres étant obtenus aux températures les plus basses. Le déterminisme génétique du caractère chez ces espèces peut être très complexe : chez $D$. melanogaster, on connaît au moins trois gènes, soumis à l'action de modificateurs (ROBERTSON et al., 1977).

En général ce polymorphisme ne s'exprime que chez les femelles et affecte un nombre de tergites plus ou moins important. Tous les travaux qui lui sont consacrés soulignent qu'il se maintient dans la nature. On peut donc se demander s'il est adaptatif (et à quoi) et rechercher les facteurs de son maintien. Des modèles basés sur l'avantage de l'hétérozygote ou sur une sélection dépendant de la fréquence ont été proposés pour expliquer le maintien au laboratoire de polymorphismes mettant en jeu des allèles plus ou moins délétères. Il est intéressant de tester si ces modèles peuvent s'appliquer à ce polymorphisme naturel.

Le matériel d'étude choisi ici est $D$. erecta (sous-groupe melanogaster), espèce facile à élever au laboratoire sur milieu au maïs ensemencé de levure vivante. Le déterminisme génétique de la coloration abdominale de cette espèce est simple : un gène diallélique porté par le chromosome $X$, l'allèle sombre $(C)$ dominant le clair $(c)$ (ce qui confirme les résultats de Ashburner puis David, commun. pers.). Le polymorphisme ne s'exprime que chez les femelles, qui sont soit claires (génotype symbolisé ici par $c / c$ ), soit sombres (génotypes $C / C$ et $C / c$ ) ; les mâles sont sombres quel que soit leur génotype (symbolisé par $C / Y$ ou $c / Y$ ). Il est possible de connaître le génotype des femelles par simple observation dans le cas des femelles de phénotype clair ou après back-cross pour celles de phénotype sombre ; celui des mâles ne peut être déterminé que par back-cross. La température d'élevage ne modifie pas l'expression phénotypique. Aucune distorsion de ségrégation n'a été mise en évidence.

$D$. erecta vit en Afrique tropicale, à une température moyenne comprise entre $22^{\circ}$ et $30^{\circ} \mathrm{C}$ (Lachaise \& Tsacas, 1974). Elle utilise les fruits de Pandanus $s p$. tombés au sol comme gîtes larvaires exclusifs, de mars à juin en Côte-d'Ivoire (Rio et al., 1983). En dehors de la période de fructification des Pandanus, de rares individus isolés ont été capturés, toujours des mâles (LAchaise, commun. pers.). Si cette espèce ne diapause pas (à l'état de pupes par exemple) pendant cette période, une telle écologie pourrait provoquer la perte par dérive (à la suite d'un goulot d'étranglement des effectifs) de l'un des deux allèles de coloration de l'abdomen, et donc du polymorphisme. Or celuici se maintient dans les populations naturelles sans que l'on ait encore précisé la fréquence des deux phénotypes. Ce maintien pourrait être lié à un mécanisme sélectif : sélection larvaire différentielle pour l'accès aux ressources et/ou sélection sexuelle, liée par exemple à un choix des mâles parmi les différents phénotypes des femelles. Un mécanisme de ce type a été recherché à l'aide de trois types d'expériences conduites à différentes températures (la coloration de la cuticule joue un rôle important dans la régulation thermique des insectes) : 
- des expériences en cages à populations, où les individus sont soumis à une très forte compétition ;

- l'analyse de la sélection s'effectuant au stade adulte ;

- la recherche de différences physiologiques entre les génotypes aux stades larvaire et adulte.

\section{Matériel et méthodes}

\section{A. Matériel}

La souche utilisée dans cette étude (220-5, Gif-sur-Yvette) a été ramenée en mars 1980 de Grand Bassam (Côte d'Ivoire). Fondée à partir de plusieurs dizaines d'individus émergés d'un même syncarpe de Pandanus, elle a été maintenue par croisements en masse ; elle peut être considérée comme isogénisée. Des souches homozygotes sombre et claire ont été extraites de cette souche à partir respectivement de 200 et 150 lignées isofemelles.

\section{B. Méthodes}

\section{Analyse de populations expérimentales}

Des cages à populations sont constituées à partir de 1600 femelles homozygotes, que l'on suppose fécondées, extraites de la souche claire ou de la souche sombre. Les fréquences initiales de l'allèle clair chez ces femelles sont $0,10,0,50$ et 0,90 . Les cages sont placées à $20^{\circ}, 22^{\circ}, 25^{\circ}$ ou $28^{\circ} \mathrm{C}$ et suivies pendant 30 générations non chevauchantes. La photopériode est de L.D.12: 12. L'humidité, toujours difficile à contrôler est assurée simplement par des cristallisoirs d'eau placés dans les étuves. La concurrence alimentaire entre les larves est forte. Pour des raisons pratiques, il n'a pas été possible de faire plusieurs répétitions de chaque cage.

A chaque génération, les adultes sont dénombrés dans chaque cage. La sélection sur les stades larvaire et adulte est estimée par la technique des blocages : une partie des œufs pondus dans la cage se développent sans apport supplémentaire de nourriture, fournissant ainsi une image de la compétition alimentaire intervenue dans la cage (blocage avec concurrence $=\mathrm{BAC}$ ) ; une quantité similaire d'œufs se développe sur un milieu alimentaire très enrichi par rapport aux conditions régnant dans la cage, supprimant ainsi toute concurrence alimentaire entre les larves (blocage sans concurren$\mathrm{ce}=\mathrm{BSC}$ ). La comparaison des fréquences obtenues en BAC et en BSC permet d'estimer la sélection larvaire; les différences de fréquence obtenues en cages aux générations $n$ et $n+1$ peuvent résulter de la sélection larvaire ou de la sélection sexuelle. Les génotypes homozygotes sombres et hétérozygotes ne peuvent être distingués qu'après back-cross. L'estimation de la fréquence de l'allèle clair chez les femelles est donc faite par la méthode du maximum de vraisemblance (ANXolabéHère, 1978) : si l'on observe A femelles sombres, B claires, et parmi les femelles sombres testées par back-cross $\mathrm{C}$ homozygotes et $\mathrm{D}$ hétérozygotes, la fréquence de l'allèle clair est :

$q=\frac{-(2 C+D)+\sqrt{(2 C+D)^{2}+8(A+B)(2 B+D)}}{4(A+B)}$ 
$\sigma_{q}^{2}=\frac{q\left(1-q^{2}\right)}{2 N(k-k q+2 q)}$ avec $k=\frac{C+D}{A}$

Lorsque la fréquence de l'allèle clair est faible, cette estimation a été faite par la formule

$q=\sqrt{\frac{4 B+1}{4 N+1}}$

Les effectifs des génotypes mâles (déterminés par back-cross) ont été comparés à ceux attendus avec la fréquence estimée des femelles claires à la génération précédente (puisque le gène est porté par le chromosome $\mathrm{X}$ ). En théorie, le gène, ne s'exprimant pas chez les mâles, ne devrait pas donner prise à la sélection.

\section{Analyse de la sélection sur le stade adulte}

Des lots de 200 femelles et 200 mâles âgés de 5 jours élevés dans les mêmes conditions sont mis en présence durant $1 \mathrm{~h} 45$ à $22^{\circ} \mathrm{C}$ dans une bouteille de milieu au maïs. Ces conditions assurent la fécondation de 20 à $75 \%$ des femelles tout en ne permettant pas de double fécondation. L'examen de la descendance de chaque femelle permet de déterminer le génotype du mâle auquel elle s'est accouplée. Les femelles hétérozygotes sont marquées par section de l'extrémité de l'aile, des expériences complémentaires ont montré que cette section est sans influence sur les résultats. L'analyse est faite pour les fréquences de l'allèle clair $0,10,0,30,0,50,0,70$ et 0,90 , les proportions des mâles et femelles mis en présence étant conformes à la loi de HARDYWeinberG. Le nombre de couples d'un type formés est comparé au nombre attendu sous l'hypothèse de croisements panmictiques par un test de $\chi^{2}$ de conformité. La comparaison de l'aptitude à l'accouplement de 2 génotypes femelles est faite par le coefficient K (PETIT, 1958) :

$\mathrm{K}_{\mathrm{f}}=\frac{\mathrm{N}_{1}}{\left(\mathrm{~N}_{2}+1\right)} \cdot \frac{\mathrm{n}_{2}}{\mathrm{n}_{1}}$

$N_{1}$ et $N_{2}$ sont les nombres respectifs de femelles claires et sombres fécondées et $n_{1}$ et $\mathrm{n}_{2}$ les nombres respectifs de femelles claires et sombres mises en compétition. En absence de sélection on attend $K_{f}=1$. L'erreur-standard sur ce coefficient est :

$S^{2}{ }_{K f}=\frac{K_{f}^{2} \cdot N}{N_{1} \cdot N_{2}}$ avec $N=N_{1}+N_{2}$

La formule de l'erreur n'est valable que pour des valeurs de $\mathrm{p}$ et $\mathrm{q}$ supérieures à 0.10 .

Le coefficient $\mathrm{K}$ met en évidence tout écart à la panmixie. La meilleure méthode détectant alors une éventuelle liaison à la fréquence de cet écart est le calcul de la droite de régression entre les rapports des effectifs des 2 génotypes mis en compétition et les rapports des nombres d'individus de ces génotypes s'étant accouplés.

\section{Différences physiologiques entre les génotypes}

\section{a. Durées de développement}

Les œufs pondus pendant une heure par des femelles âgées de cinq jours et élevées dans les mêmes conditions sont dénombrés puis mis à développer à $20^{\circ}, 22^{\circ}, 25^{\circ}$ ou 
$28^{\circ} \mathrm{C}$. Les œufs non éclos sont dénombrés toutes les heures. Les larves de premier stade obtenues à chaque température se développent en absence de compétition alimentaire. Les durées de vie larvo-nymphale de chaque génotype sont estimées en notant toutes les heures les émergences des adultes et comparées par des tests $t$.

\section{b. Fécondité des femelles}

$24 \mathrm{~h}$ après leur naissance, une femelle et deux mâles sont placés dans un tube de milieu à $22^{\circ}$ ou $25^{\circ} \mathrm{C}$. Toutes les $48 \mathrm{~h}$ jusqu'à la mort de la femelle, les mouches sont transférées dans un tube frais puis les adultes, éclos en absence de compétition larvaire, sont dénombrés. Dix répétitions au moins sont faites pour chaque génotype à chaque température. Les pontes des quatre génotypes aux $15^{\mathrm{e}}$ et respectivement $30^{\mathrm{e}}$ et $26^{\mathrm{e}}$ jours à $22^{\circ}$ et $25^{\circ} \mathrm{C}$ sont comparées par une analyse de variance.

\section{c. Longévité des adultes}

Dix mâles ou 10 femelles de chaque génotype sont placés dès l'émergence en tubes à $20^{\circ}, 22^{\circ}, 25^{\circ}$ ou $28^{\circ} \mathrm{C}$. Le nombre d'individus morts dans chaque tube est noté toutes les $24 \mathrm{~h}$ jusqu'à l'extinction de la population du tube. Les tubes de milieu sont renouvelés toutes les semaines. Trois répétitions de chaque expérience sont faites. Les durées de vie moyennes de chaque génotype sont comparées par une analyse de variance à deux facteurs.

\section{Résultats}

\section{A. Evolution de la fréquence allélique en cages à population}

L'évolution de la fréquence de l'allèle clair dans les cages suivies à $20^{\circ}$ et $22^{\circ} \mathrm{C}$ est représentée sur la figure $1 \mathrm{~A}$, celle des cages suivies à $25^{\circ}$ et $28^{\circ} \mathrm{C}$ sur la figure $1 \mathrm{~B}$. L'effectif des adultes dénombrés à chaque génération est le plus souvent proche de 1500 animaux à $22^{\circ}$ et $25^{\circ} \mathrm{C}$ et de 1000 animaux à $20^{\circ}$ et $28^{\circ} \mathrm{C}$.

Les évolutions en cage sont différentes selon la température : à $20^{\circ} \mathrm{C}$ (fig. $1 \mathrm{~A}$ ) on observe une tendance à la fixation, de l'allèle clair si la fréquence initiale est élevée $(0,90)$, de l'allèle sombre si elle est moyenne $(0,50)$; à $22{ }^{\circ} \mathrm{C}$ (fig. $1 \mathrm{~A}$ ) la fréquence de l'allèle clair s'approche de 0,20 lorsque la fréquence initiale est 0,10 ou 0,50 , et atteint 0,50 lorsque la fréquence initiale est 0,90 . A $25^{\circ}$ et $28^{\circ} \mathrm{C}$ (fig. $1 \mathrm{~B}$ ) l'allèle clair disparaît lorsque la fréquence initiale est basse, reste proche de sa fréquence initiale lorsqu'elle est intermédiaire et tend à se fixer si elle est élevée : à ces deux températures la probabilité de fixation des deux allèles ne dépend que de la fréquence initiale.

Les $\chi^{2}$ entre les effectifs des femelles claires observées dans les cages à populations et dans les BAC issus de la même population parentale ne sont pas significatifs (ils ne sont donc pas présentés ici), les BAC peuvent donc être considérés comme un échantillon de la cage dont ils sont extraits.

Les fréquences génotypiques des femelles et des mâles dans les BSC et les BAC issus de chaque cage ne sont pas significativement différentes des fréquences attendues sous l'hypothèse de HARDY-WeInBERG. Tout se passe comme s'il n'y avait pas de forte sélection larvaire ni sexuelle à l'échelle d'une génération. 


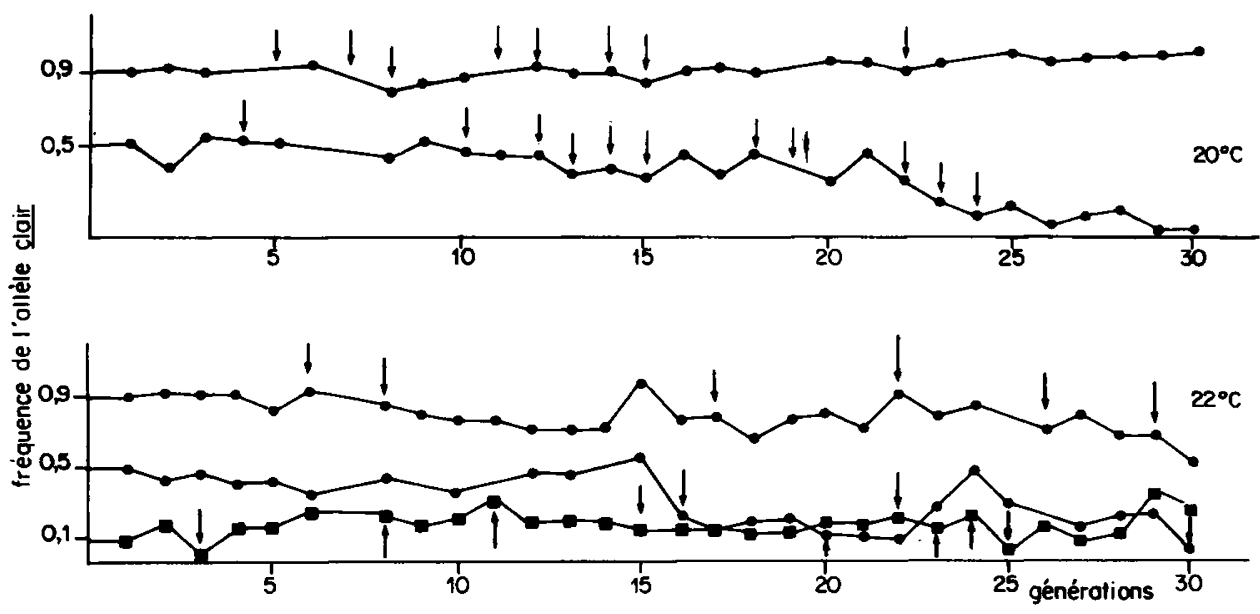

FIG. $1 \mathrm{~A}$

Variations de la fréquence de l'allèle clair au cours des générations dans les cages maintenues à 20 ou $22{ }^{\circ} \mathrm{C}$.

Gene-frequency changes in population cages at 20 or $22^{\circ} \mathrm{C}$.

Les flèches indiquent les générations où moins de 350 femelles ont émergé, les astérisques celles où moins de 100 femelles ont émergé. La fréquence allélique a été calculée par la méthode du maximum de vraisemblance lorsque les back-cross ont pu être réalisés, par la racine carrée de la fréquence des femelles claires dans le cas contraire.

Arrows and stars respectively point to generations where less than 350 and 100 females hatched. The allelic frequency was calculated either from back-cross whenever possible, or by the square root of the frequency of the light females.

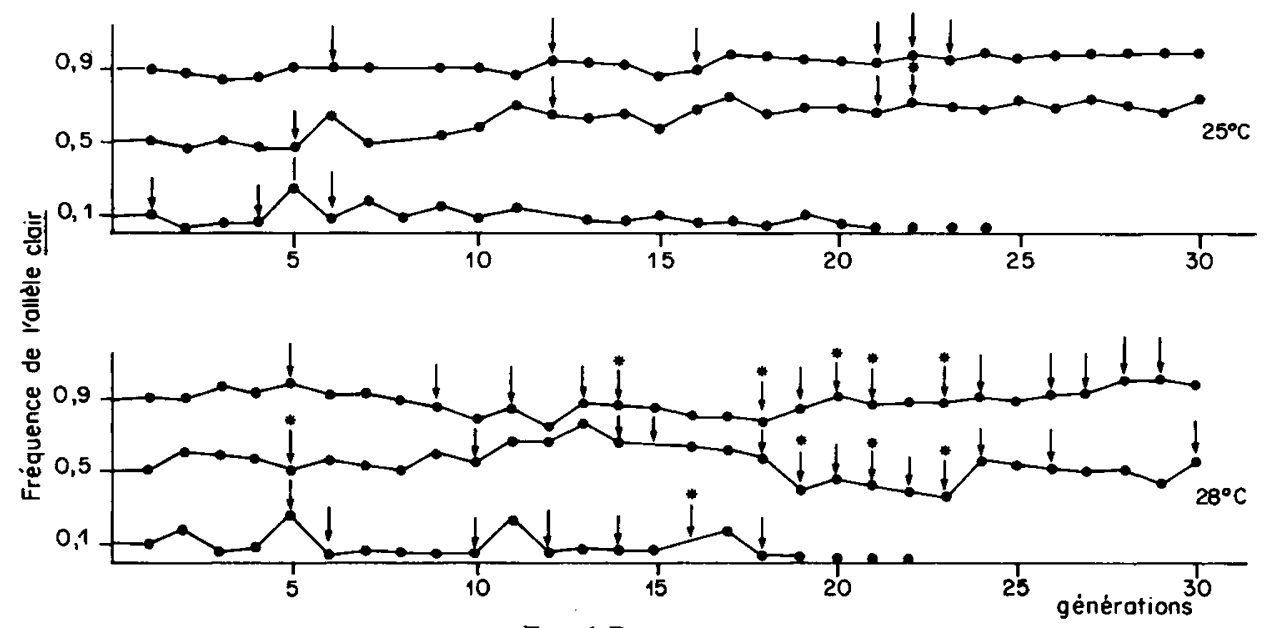

Fig. $1 \mathrm{~B}$

Variations de la fréquence de l'allèle clair au cours des genérations dans les cages maintenues à 25 ou $28^{\circ} \mathrm{C}$.

Gene-frequency changes in population cages at 25 or $28^{\circ} \mathrm{C}$.

Même légende qu'en figure $1 \mathrm{~A}$.

Same caption than in figure $1 \mathrm{~A}$. 
Une sélection larvaire dépendant de la fréquence a été recherchée en calculant l'équation des droites de régression entre les rapports des effectifs des femelles claires et sombres écloses dans les BSC et les BAC (après transformation logarithmique des données, tableau 1). Les points correspondant aux différentes cages étudiées à une même température ont été regroupés pour augmenter la variation en abscisse. La pente de ces droites n'est significativement différente de la bissectrice qu'à $20^{\circ} \mathrm{C}$, température à laquelle une sélection larvaire dépendant de la fréquence pourrait intervenir.

\section{TABLEAU 1}

Equations des droites de régression entre les rapports des effectifs des femelles claires et sombres dans les BSC et les BAC (Y) et valeurs des tests des pentes de ces droites par rapport à la bissectrice $\left(t_{h}\right)$ selon la température de maintien des cages

Equations of the regression lines of light vs dark females in BSC and BAC (Y) and comparison of slopes with the diagonal $\left(t_{h}\right)$ for each cage's temperature

\begin{tabular}{c|c|cc}
\hline \hline $\begin{array}{c}\text { Température } \\
\left({ }^{\circ} \mathrm{C}\right)\end{array}$ & $\mathrm{Y}$ & \multicolumn{2}{|c}{$\mathrm{t}_{\mathrm{b}}$} \\
\hline 20 & $\mathrm{Y}=0,102+0,783 \mathrm{X}$ & $3,60^{* * *}$ & $(35 \mathrm{dd} 1)$ \\
22 & $\mathrm{Y}=-0,058+0,879 \mathrm{X}$ & 1,66 & $(61 \mathrm{dd} 1)$ \\
25 & $\mathrm{Y}=0,058+0,966 \mathrm{X}$ & 0,58 & $(51 \mathrm{dd} 1)$ \\
28 & $\mathrm{Y}=-0,011+1,029 \mathrm{X}$ & 0,64 & $(47 \mathrm{dd} 1)$ \\
\hline
\end{tabular}

$(* * *: p<0,001)$.

\section{B. Réponse à la compétition durant la vie adulte}

L'expression du polymorphisme de coloration abdominale de $D$. erecta étant limitée au sexe femelle, son maintien par l'action de la sélection sexuelle doit être envisagé. Les résultats des expériences de choix multiples sont présentés dans le tableau 2 . Chez les mâles aucun $\chi^{2}$ n'est significatif, ce qui suggère une absence de sélection sexuelle dans ce sexe. Chez les femelles les valeurs du $\chi^{2}$ significatives aux fréquences 0,50 et 0,70 témoignent d'une sélection sexuelle. A ces fréquences les coefficients de sélection sexuelle des femelles claires $(c / c)$ par rapport aux hétérozygotes $(C / c)$ significativement supérieurs à un mettent en évidence un avantage des premières sur les secondes. A la fréquence 0,90 le coefficient $\mathrm{K}_{\mathrm{f}}$ des $c / c$ par rapport aux homozygotes sombres $(C / C)$ est significativement inférieur à un. Cet avantage des $C / c$ est probablement dû à un biais lié au fait que le très faible nombre de femelles $C / C$ mises en compétition $(2 \%)$ a toujours été fécondé. L'hypothèse d'une sélection sexuelle dépendant de la fréquence a été testée par la comparaison à la bissectrice des pentes des droites de régression entre les femelles mises en présence et les femelles accouplées : elles n'en diffèrent pas significativement, les femelles s'accouplent donc indépendamment de leur génotype.

\section{Différences physiologiques entre les génotypes}

\section{Durées de développement}

En l'absence de compétition les mortalités des œufs, des larves et des nymphes ne sont pas significativement différentes selon le génotype (tableau 3). La durée de 


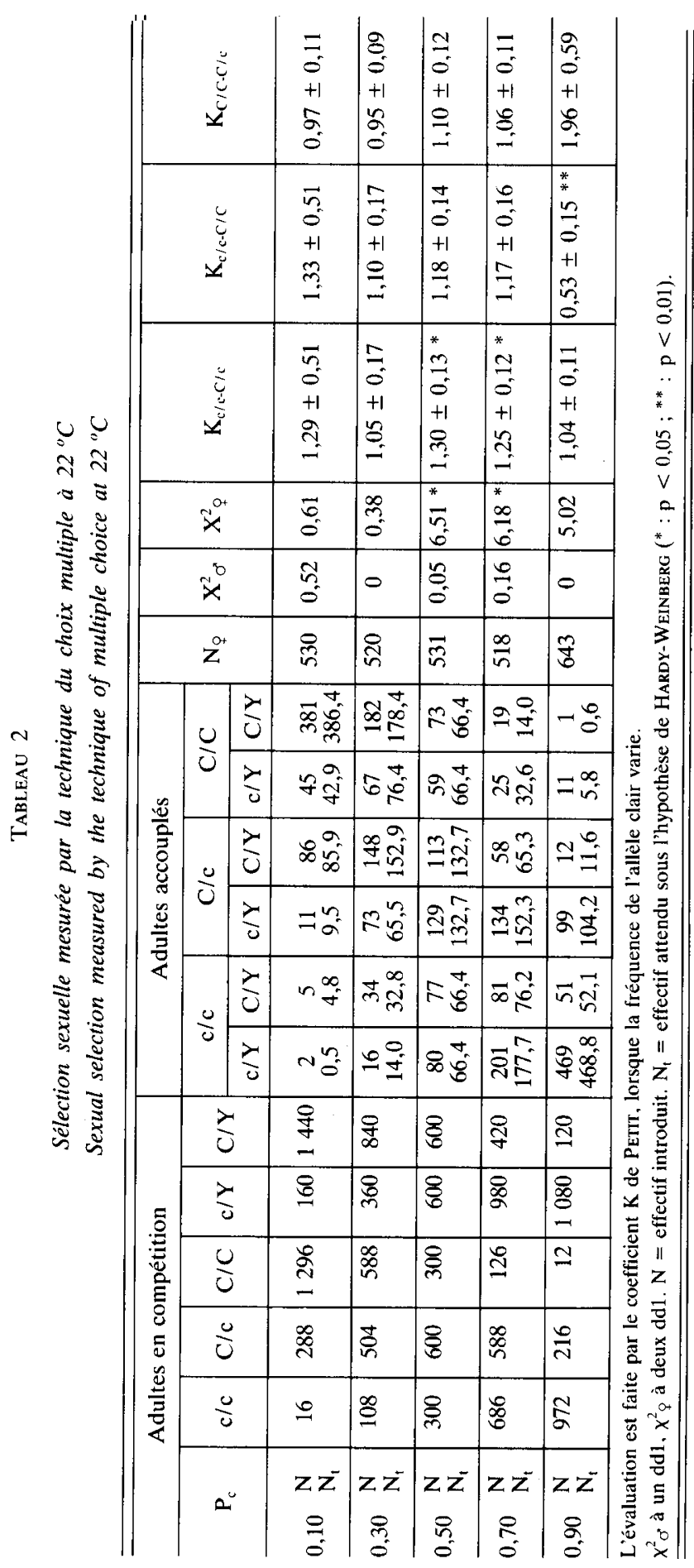




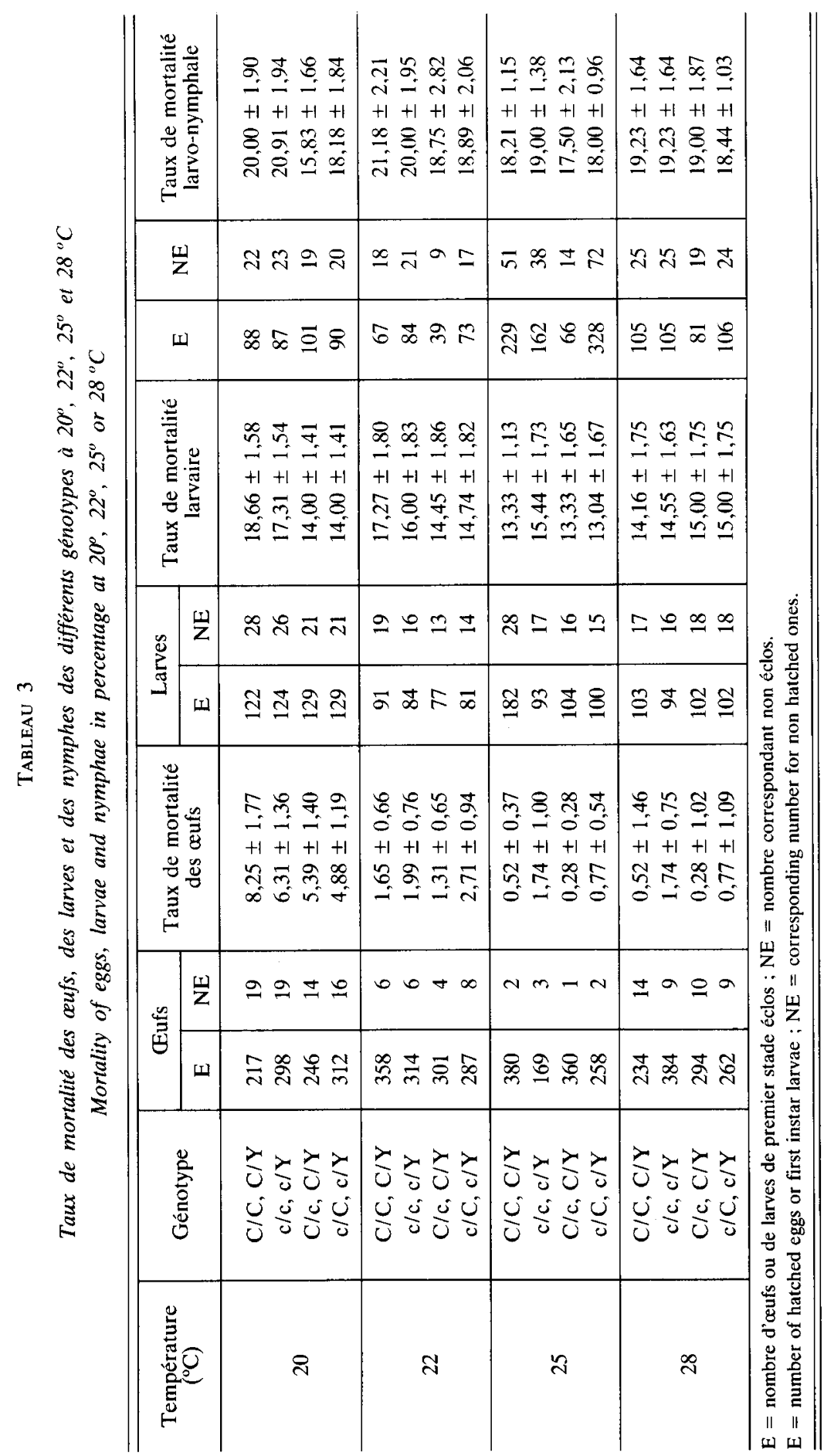


TABLEAU 4

Durée moyenne de développement larvo-nymphal des différents génotypes à $22^{\circ}, 25^{\circ}$ et $28^{\circ} \mathrm{C}$ et nombre cumulé d'adultes éclos des oeufs pondus aux $15^{e}$ et respectivement aux $30^{\circ}$ et $26^{e}$ jours à 22 et $25^{\circ} \mathrm{C}$ (a). Analyse de variance correspondante (b)

Mean larvo-nymphal developmental times of genotypes at $22^{\circ}, 25^{\circ}$ and $28^{\circ} \mathrm{C}$ and cumulated number of hatched adults from eggs laid on the $15^{\text {th }}$ and respectively $30^{\text {th }}$ and $26^{\text {th }}$ days at 22 and $25^{\circ} \mathrm{C}(\mathrm{a})$. Corresponding variance analysis (b)

\begin{tabular}{|c|c|c|c|c|c|c|}
\hline $\begin{array}{c}\text { Température } \\
\left({ }^{\circ} \mathrm{C}\right)\end{array}$ & Génotype & NL & DLN & $\boldsymbol{\sigma}$ & PC15 & $\mathrm{PC} 30 / 26$ \\
\hline 22 & $\begin{array}{l}\mathrm{C} / \mathrm{C}, \mathrm{C} / \mathrm{Y} \\
\mathrm{c} / \mathrm{c}, \mathrm{c} / \mathrm{Y} \\
\mathrm{C} / \mathrm{c}, \mathrm{C} / \mathrm{Y} \\
\mathrm{c} / \mathrm{C}, \mathrm{c} / \mathrm{Y}\end{array}$ & $\begin{array}{r}229 \\
162 \\
66 \\
328\end{array}$ & 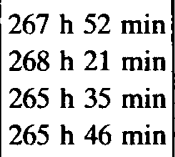 & $\begin{array}{l}45 \mathrm{~min} \\
57 \mathrm{~min} \\
50 \mathrm{~min} \\
50 \mathrm{~min}\end{array}$ & $\begin{array}{l}184 \pm 25 \\
158 \pm 40 \\
237 \pm 44 \\
197 \pm 35\end{array}$ & $\begin{array}{l}308 \pm 41 \\
292 \pm 38 \\
462 \pm 52 \\
341 \pm 45\end{array}$ \\
\hline 25 & $\begin{array}{l}\mathrm{C} / \mathrm{C}, \mathrm{C} / \mathrm{Y} \\
\mathrm{c} / \mathrm{c}, \mathrm{c} / \mathrm{Y} \\
\mathrm{C} / \mathrm{c}, \mathrm{C} / \mathrm{Y} \\
\mathrm{c} / \mathrm{C}, \mathrm{c} / \mathrm{Y}\end{array}$ & $\begin{array}{l}67 \\
84 \\
39 \\
73\end{array}$ & $\mid \begin{array}{l}228 \text { h } 02 \text { min } \\
228 \text { h } 37 \text { min } \\
228 \text { h } 05 \text { min } \\
228 \text { h } 14 \text { min }\end{array}$ & $\begin{array}{l}38 \mathrm{~min} \\
35 \mathrm{~min} \\
43 \mathrm{~min} \\
39 \mathrm{~min}\end{array}$ & $\begin{array}{l}224 \pm 37 \\
273 \pm 26 \\
294 \pm 29 \\
300 \pm 42\end{array}$ & $\begin{array}{l}313 \pm 30 \\
350 \pm 55 \\
347 \pm 28 \\
356 \pm 30\end{array}$ \\
\hline 28 & $\begin{array}{l}\mathrm{C} / \mathrm{C}, \mathrm{C} / \mathrm{Y} \\
\mathrm{c} / \mathrm{c}, \mathrm{c} / \mathrm{Y} \\
\mathrm{C} / \mathrm{c}, \mathrm{C} / \mathrm{Y} \\
\mathrm{c} / \mathrm{C}, \mathrm{c} / \mathrm{Y}\end{array}$ & $\begin{array}{r}88 \\
87 \\
101 \\
90\end{array}$ & $\begin{array}{l}190 \text { h } 41 \mathrm{~min} \\
191 \text { h } 41 \mathrm{~min} \\
190 \text { h } 09 \mathrm{~min} \\
190 \text { h } 17 \mathrm{~min}\end{array}$ & $\begin{array}{r}1 \text { h } 17 \text { min } \\
42 \mathrm{~min} \\
45 \mathrm{~min} \\
1 \text { h } 15 \mathrm{~min}\end{array}$ & & \\
\hline
\end{tabular}

$\mathrm{NL}=$ nombre de larves. $\mathrm{DLN}=$ durée moyenne de développement larvo-nymphal. $\sigma=$ écart-type. $\mathrm{PC} 15=$ ponte cumulée au $15^{\mathrm{c}}$ jour. $\mathrm{PC} 30=$ ponte cumulée au $30^{\circ}$ jour $\left(22^{\circ} \mathrm{C}\right)$ ou au $26^{\mathrm{c}}$ jour $\left(25^{\circ} \mathrm{C}\right)$.

\begin{tabular}{c|c|c}
\hline \hline $\mathrm{F}$ & $15^{\mathrm{e}}$ jour & $\left.\begin{array}{l}26^{\mathrm{e}} \\
30^{\mathrm{e}}\end{array}\right]$ jour \\
\hline $22^{\circ} \mathrm{C}$ & $\begin{array}{c}10,96^{* * *} \\
(52 \mathrm{dd} 1)\end{array}$ & $\begin{array}{c}21,86^{* * *} \\
(31 \mathrm{dd} 1)\end{array}$ \\
\hline $25^{\circ} \mathrm{C}$ & $\begin{array}{c}7,16 \\
(31 \mathrm{dd} 1)\end{array}$ & $\begin{array}{c}1,18 \\
(23 \mathrm{dd} 1)\end{array}$ \\
\hline${ }^{* * *}=\mathrm{p}<0,001$.
\end{tabular}

développement larvo-nymphal des femelles $c / c$ est significativement supérieure à celle des femelles $c / C$ à $22^{\circ} \mathrm{C}$ (tableau $4 ; \mathrm{t}=2,00$ ). Le gène ne s'exprimant que chez les femelles, une certaine dilution des différences entre génotypes peut se produire du fait de la présence des mâles dans ces expériences si ces derniers ne donnent pas prise à la sélection, comme c'est probable.

\section{Fécondité des femelles}

Parmi les femelles pondeuses certaines sont stériles, mais cette stérilité est indépendante de leur génotype. Les nombres cumulés des adultes éclos des œufs pondus par les femelles des différents génotypes à 22 et à $25^{\circ} \mathrm{C}$ aux $15^{\mathrm{e}}$ et respectivement $30^{\mathrm{e}}$ ou $26^{\mathrm{e}}$ jours sont portés sur le tableau 4. L'analyse de variance correspondante met en évidence une différence selon le génotype à $22^{\circ} \mathrm{C}$ ainsi qu'à $25^{\circ} \mathrm{C}$ au $15^{\mathrm{e}}$ jour, cette 
différence disparaissant à $25^{\circ} \mathrm{C}$ au $26^{\mathrm{e}}$ jour. A $22^{\circ} \mathrm{C}$ les descendants des femelles $\mathrm{c} / \mathrm{c}$ sont significativement moins nombreux que ceux des autres génotypes et ceux des femelles $C / c$ significativement plus nombreux, ces différences subsistant tout au long de la vie des femelles. A $25^{\circ} \mathrm{C}$ au $15^{\mathrm{e}}$ jour les descendants des femelles $C / C$ sont moins nombreux que ceux des autres génotypes mais cette infériorité a disparu à la fin de la vie des femelles pondeuses, au $26^{\mathrm{c}}$ jour. Les œufs s'étant développés en présence d'un excès de nourriture, il est peu probable que la compétition alimentaire ait influencé ces résultats.

\section{Longévité des adultes}

L'analyse de variance à deux facteurs comparant les longévités moyennes des adultes (tableau 5) détecte une différence selon la température d'élevage et, chez les femelles, selon le génotype. A $20^{\circ} \mathrm{C}$ les femelles $c / c$ ont la durée de vie moyenne la plus longue, $\mathrm{C} / \mathrm{C}$ à 22 et $25^{\circ} \mathrm{C}$ et $\mathrm{C} / \mathrm{c}$ à $28^{\circ} \mathrm{C}$. La durée de vie moyenne des mâles est la même pour les deux génotypes à chaque température ; elle est inférieure à celle des femelles à $20^{\circ}, 22^{\circ}$ et $25^{\circ} \mathrm{C}$ mais nettement supérieure à $28^{\circ} \mathrm{C}$.

TABleaU 5

Durée de vie moyennes des adultes mâles et femelles des différents génotypes (a) et analyse de variance correspondante (b) à $20,22,25$ et $28^{\circ} \mathrm{C}$

Mean life spans of males and females of various genotypes (a) and relevant variance analysis (b) at $20,22,25$ and $28^{\circ} \mathrm{C}$

\begin{tabular}{l|c|c|c|c|c|c|c|c}
\hline \multirow{2}{*}{ Génotype } & \multicolumn{2}{|c|}{$20^{\circ} \mathrm{C}$} & \multicolumn{2}{c|}{$22^{\circ} \mathrm{C}$} & \multicolumn{2}{c|}{$25^{\circ} \mathrm{C}$} & \multicolumn{2}{c}{$28{ }^{\circ} \mathrm{C}$} \\
\cline { 2 - 9 } & DVA & $\sigma$ & DVA & $\sigma$ & DVA & $\sigma$ & DVA & $\sigma$ \\
\hline \multirow{2}{*}{$\mathrm{C} / \mathrm{C}$} & 48,52 & 5,32 & 42,30 & 3,79 & 23,07 & 1,89 & 23,83 & 1,94 \\
$\mathrm{c} / \mathrm{c}$ & 59,96 & 4,79 & 30,97 & 2,84 & 21,37 & 1,58 & 19,19 & 1,87 \\
$\mathrm{C} / \mathrm{c}$ & 44,55 & 4,67 & 33,76 & 3,71 & 17,72 & 1,53 & 25,35 & 2,03 \\
$\mathrm{c} / \mathrm{C}$ & 43,50 & 3,92 & 36,97 & 3,47 & 21,06 & 2,28 & 23,02 & 2,32 \\
\hline \multirow{2}{*}{$\sigma^{*} \mathrm{C} / \mathrm{Y}$} & 26,56 & 2,53 & 28,13 & 2,53 & 18,79 & 1,48 & 28,47 & 2,34 \\
$\mathrm{c} / \mathrm{C}$ & 26,30 & 5,88 & 30,66 & 2,81 & 20,14 & 1,70 & 26,34 & 2,27 \\
\hline
\end{tabular}

DVA $=$ durée moyenne de vic adulte. $\sigma=$ écart-type.

\begin{tabular}{l|l|c|c}
\hline \hline & \multicolumn{1}{|c|}{ Variation } & F & dd1 \\
\hline \multirow{4}{*}{ Femelles } & Génotype & $11,90^{* *}$ & 3 \\
& Température & $57,10^{* *}$ & 3 \\
& Interaction & 1,42 & 9 \\
& Résiduelle & - & 32 \\
\hline \multirow{4}{*}{ Mâles } & Génotype & 0.43 & 1 \\
& Température & $5,01^{* *}$ & 3 \\
& Interaction & 0,21 & 3 \\
& Résiduelle & - & 16 \\
\hline
\end{tabular}

${ }^{* *}: \mathrm{p}<0,01$. 


\section{Discussion et conclusion}

Les méthodes utilisées ici sont très classiques et ont permis de mettre en évidence les facteurs du maintien du polymorphisme dans de nombreux cas, que ce polymorphisme soit phénotypique ou enzymatique. Les évolutions de la fréquence allélique dans les cages à populations suivies à quatre températures différentes à partir de trois fréquences initiales sont différentes selon la température d'élevage. Mais aucune sélection sur les stades larvaire ou adulte n'a été mise en évidence ici, sauf peut-être à $20^{\circ} \mathrm{C}$ où on ne peut exclure l'existence d'une sélection larvaire avantageant les femelles sombres. Certaines des expériences complémentaires réalisées hors des cages à populations semblent indiquer l'existence d'une sélection, d'autes favorisent une interprétation neutraliste du maintien du polymorphisme abdominal chez $D$. erecta. Les résultats obtenus chez les mâles sont tous concordants : les mâles ne donnent pas prise à la sélection, ce qui est en accord avec la théorie puisque le polymorphisme ne s'exprime dans cette espèce que chez les femelles.

Chez ces dernières les arguments en faveur de la neutralité du gène de coloration abdominale sont les suivants: les variations en cages à populations de la fréquence allélique ne sont pas différentes de celles que l'on observerait sous l'effet du hasard, sauf peut-être à $20^{\circ} \mathrm{C}$, et la fréquence allélique dans les blocages, avec et sans concurrence, n'est pas significativement différente de celle que l'on attend sous l'hypothèse de Hardy-Weinberg. Il faut noter que la température de $20^{\circ} \mathrm{C}$ est la plus éloignée de la température moyenne de l'habitat naturel de $D$. erecta (comprise entre 22 et $28^{\circ} \mathrm{C}$ ). Les mortalités des œufs, larves et nymphes des différents génotypes sont les mêmes, ainsi que leurs durées de développement. Il n'y a pas de sélection sexuelle à $22^{\circ} \mathrm{C}$ lorsque la fréquence de l'allèle clair est $0,10,0,30$ ou 0,90 . Jusqu'à présent la théorie neutraliste n'a été invoquée que pour rendre compte de polymorphismes biochimiques. Par exemple YAMAZAKI (1971) interprète le maintien des différents allèles de l'estérase- 5 en cage à population chez $D$. pseudoobscura à l'aide de cette théorie (le gène est porté par le chromosome $\mathrm{X}$ ). Mais les fréquences alléliques varient ici beaucoup plus d'une génération à l'autre et au cours du temps que celles observées par YAMAZAKı : en 30 générations l'allèle clair est éliminé dans quatre cages sur 11 et le sombre dans trois cages; de plus il n'est pas rare d'observer des variations de fréquence de l'ordre de 0,20 d'une génération à la suivante, parfois en l'absence de diminution concomitante d'effectifs.

Les arguments en faveur de l'intervention de la sélection sont les suivants : dans les cages à $20^{\circ} \mathrm{C}$ l'intervention d'une sélection larvaire ne peut être exclue, l'allèle sombre étant avantagé par cette sélection. Le développement larvo-nymphal des femelles $c / c$ est plus long que celui des femelles $c / C$ à $22^{\circ} \mathrm{C}$ (dans les deux cas les mâles sont $c / Y$ et leur présence n'interfère donc pas avec ces résultats). Une sélection sexuelle avantageant les femelles $c / c$ par rapport aux $C / c$ a été mise en évidence à $22{ }^{\circ} \mathrm{C}$ lorsque la fréquence de l'allèle clair est 0,50 et 0,70 . Les femelles $c / c$ pondent moins et les femelles $C / c$ plus à $22^{\circ} \mathrm{C}$ que les femelles des autres génotypes. La durée de vie des adultes varie selon leur génotype, le génotype à durée de vie la plus longue différant selon la température d'élevage.

Ces arguments en faveur de la sélection sont relativement contradictoires : aucun allèle ou génotype n'est systématiquement avantagé ou désavantagé et lorsqu'un avantage a pu être mis en évidence il était toujours faible. Il semble donc que l'on puisse 
conclure de cette étude que le gène de coloration abdominale chez $D$. erecta se comporte comme un gène neutre au laboratoire.

Reçu le 27 janvier 1986.

Accepté le 7 avril 1987.

\section{Remerciements}

Les conseils amicaux et la compétence de Madame C. Petrt et de D. Anxolabéhère dans ce genre d'études m'ont permis de mener ce travail à son terme, malgré des résultats rarement encourageants. J. David, D. Lachaise et M. Veuille ont eu la patience de relire le manuscrit de cet article ; leurs remarques, ainsi que celles des trois lecteurs de Génétique Sélection Evolution en ont grandement amélioré la version finale.

\section{Références bibliographiques}

ANXolABÉHÈre D., 1978. Analyse expérimentale et théorique du rôle des valeurs sélectives variables dans le maintien du polymorphisme au locus sepia chez Drosophila melanogaster. Thèse de Doctorat d'Etat, Université Paris 7.

Basden E.B., 1957. Japanese Drosophilidae (Dipt.) : a review. Ent. Mon. Mag., 93, 208-211.

Dobzhansky T., Pavan C., 1943. Studies on Brazilian species of Drosophila. Bol. Fac. Filos. Cienc. Letr. Univ. S. Paulo, 36 (4), 7-72.

Lachaise D., Tsacas L., 1974. Les Drosophilidae des savanes préforestières de la région tropicale de Lamto (Côte-d'Ivoire). 2: Le peuplement des fruits de Pandanus candelabrum (Pandanacée). Ann. Univ. Abidjan, 7, 153-192.

Oshima C., 1952. Genetic analysis of the dimorphism of color pattern in D. rufa. Drosophila Inf. Serv., 26, 116.

PetrT C., 1958. Le déterminisme génétique et psychophysiologique de la compétition sexuelle chez Drosophila melanogaster. Bull. Biol. Fr. Belg., 92, 248-329.

Rio B., Couturier G., Lemeunier F., Lachaise D., 1983. Evolution d'une spécialisation saisonnière chez Drosophila erecta (Dipt. Drosophilidae). Ann. Soc. Ent. Fr., 19, 235-248.

Robertson A., Briscoe D.A., Louw J.H., 1977. Variation in abdomen pigmentation in Drosophila melanogaster females. Genetica, 47, 73-76.

YAmAZAKI T., 1971. Measurement of fitness at the esterase-5 locus in Drosophila pseudoobscura. Genetics, 67, 579-603. 